\section{Which Triptan?- Opportunity for Same or Better Outcomes at Lower Cost}

With 7 triptans on the market in the United States, there is considerable opportunity for managed care plans to negotiate significant price reductions from manufacturers for preferred formulary status, particularly under 3-tier benefit designs or in closed formularies (i.e., 100\% member cost-share for nonformulary drugs). Last year, the Department of Defense and Veterans Affairs released a request for (price) proposal from manufacturers of 4 triptans (the newest triptan, eletriptan [Relpax], was apparently introduced after the RFP was prepared). The drugs under consideration by DOD/VA were almotriptan (Axert), sumatriptan (Imitrex), rizatriptan (Maxalt), and zolmitriptan (Zomig), based, in part, on prices bid in an RFP in October 2002. The RFP found the 4 triptans to be therapeutically equivalent, based on "similar outcomes, similar side-effect profiles, and sufficient safety data." Naratriptan (Amerge) and frovatriptan (Frova) "should not be considered front-line agents" according to the VA "because of less favorable pain-free results at two hours as compared to the other triptans." On April 7, 2003, the comptroller general denied a protest from the manufacturer of sumatriptan that claimed "discrepancies in the meta-analyses" and alleged superiority of sumatriptan; the comptroller general said, "Even accepting that sumatriptan could be considered superior, the agency's determination that the differences between the drugs were not material for its purposes has not been shown to be unreasonable."

In a previous article in this Journal, a meta-analysis showed that the number needed to treat to achieve 1 patient pain free at 2 hours was 3.2 patients for rizatriptan $10 \mathrm{mg}, 4.2$ patients for zolmitriptan $5 \mathrm{mg}, 4.7$ patients for either almotriptan $12.5 \mathrm{mg}$ or sumatriptan $100 \mathrm{mg}$, and 5.9 patients for sumatriptan $50 \mathrm{mg}$. Naratriptan $2.5 \mathrm{mg}$ required 8.2 patients, and frovatriptan $2.5 \mathrm{mg}$ required 11.3 patients treated to achieve 1 patient pain free at 2 hours. $^{2}$ In this issue of the Journal, Williams and Reeder found in their base-case analysis that the average cost-effectiveness ratios, using March 2004 prices, were \$82, \$133, and \$138 per composite end point defined as Sustained pain-free and No Adverse Events (SNAE) for migraine attacks for almotriptan $12.5 \mathrm{mg}$, sumatriptan $50 \mathrm{mg}$, and sumatriptan $100 \mathrm{mg}$, respectively. ${ }^{3}$ In other words, sumatriptan had a price premium of $62 \%$ to $68 \%$ compared with almotriptan. The incremental cost-effectiveness ratios for almotriptan $12.5 \mathrm{mg}$ were $\$ 12$ and $\$ 16$ (compared with sumatriptan $50 \mathrm{mg}$ and sumatriptan $100 \mathrm{mg}$, respectively) per incremental attack at which SNAE is achieved. This research has significant value for managed care pharmacists by determining cost for the combined outcomes of efficacy and safety. Readers should note that this analysis by Williams and Reeder included the 67\% price increase for almotriptan (from $\$ 10.99$ average wholesale price per unit to $\$ 18.44$ per unit) that was imposed in late 2003 when the drug was transferred from one manufacturer to another.

\section{Does Member Cost Sharing Pose a Threat to Desirable Patient Outcomes?}

Cost sharing by health plan beneficiaries is on the way up, both in dollar amounts or percentages and in the proportion of beneficiaries affected by cost sharing. In 2003, 96\% of workers and dependents with health benefits sponsored by employers had either copay or coinsurance requirements for medical office visits, $92 \%$ were required to contribute to payment of the monthly family premium, 63\% had 3-tier cost sharing for prescription drugs (up from 55\% in 2002, 42\% in 2001, and 27\% in 2000), ${ }^{4}$ $79 \%$ had an annual deductible, $76 \%$ were required to contribute to the monthly premium for individual (single) coverage, $44 \%$ had a separate hospital deductible, ${ }^{5}$ and $8 \%$ had a separate prescription drug deductible. ${ }^{6}$

Research published in late 2003 claimed that the introduction of 3-tier copay designs for prescription drug benefits resulted in patients discontinuing drug therapy. ${ }^{7}$ However, careful examination of the data in that study showed mixed and even contradictory results, including the finding that the discontinuation rate for angiotensin-converting enzyme (ACE) inhibitors was twice as high in the comparison (control) group compared with one of the employer 3-tier plans in the study. ${ }^{8}$ Wogen and Frech pointed out that there is, in fact, no consensus regarding the impact of patient copay on therapy persistence and adherence. ${ }^{9}$

In this issue of the Journal, Meissner, Moore, Shinogle, Reeder, and Little found that an average \$10 (47\%) increase in copayment per prescription in a 3-tier drug benefit design for a public employer was associated with no statistically significant change in drug utilization per patient for 2 classes of drugs used to treat allergic rhinitis. ${ }^{10}$ The actual average copayment increase was $\$ 10.98$ (71\%) for nasal steroids (NSs), which was associated with an $11.3 \%$ decrease in utilization of NSs, primarily the result of a $10.2 \%$ decrease in the number of users of NSs in the year following the copay increase. However, the number of NS prescriptions per patient per year was unchanged at 2.05 versus 2.07 in the year prior to the copay increase $(P=0.842)$.

The combined utilization of low-sedating antihistamines (LSAs) and NS prescriptions increased by $8.9 \%$ following the increase in copayments for these 2 therapeutically interchangeable drugs for allergic rhinitis. Rather than causing a reduction in the utilization of LSAs, the imposition of a 3-tier copay drug benefit design and an average $\$ 7.23$ (41\%) increase in copayment per prescription was associated with a $14.8 \%$ increase in the use of LSAs, including an $11.8 \%$ increase in the number of patients using LSAs. The number of LSA prescriptions per patient per year was unchanged at 2.68 in the year following the increase in copayment compared with 2.61 in the year prior to the copay increase; $P=0.429$. While not adversely affecting patient use of drugs to treat allergic rhinitis, implementation of the increase in copayment in the 3-tier drug benefit design was associated with the intended outcome of producing cost savings for the health plan. Health plan savings were $16.3 \%$ per patient, and these health plan savings would have been larger if the costs had been adjusted for inflation. The health plan costs for all drugs for these allergic rhinitis patients fell by $13 \%$, also understated since the costs were not adjusted for inflation. 